\title{
Caso de empresa Simplemail: el dilema de la expansión de la cartera de clientes
}

\author{
Juan Pablo Torres \\ Facultad Economía y Negocios \\ Universidad de Chile \\ jtorresc@fen.uchile.cl
}

Patricio Morales

Ingeniero Comercial

Facultad Economía y Negocios

Universidad de Chile

Juan Pablo Vidal

Ingeniero Comercial

Facultad Economía y Negocios

Universidad de Chile

Peter van Eijck

Ingeniero Comercial

Facultad Economía y Negocios

Universidad de Chile

\section{Introducción}

Simplemail es una empresa inmersa en el rubro de la correspondencia que nace en 1995, se ubica y mantiene hasta la actualidad en la ciudad de Santiago, Región Metropolitana. La empresa es liderada por su dueño Pablo Rodríguez (CEO), quien 
toma las decisiones estratégicas de la empresa y es apoyado en las actividades diarias por su madre, hermano y cuñada. Hasta la fecha Simplemail tiene cuatro clientes (MTS, Punto Maestro, SCB, Construplaza), los que cubre a tiempo y de manera personalizada.

En junio de 2013 Pablo Rodríguez se ve enfrentado a un déficit de capacidad para ampliar su cartera de clientes, ya que tras algunos intentos de ampliarla tuvieron grandes problemas en el servicio que otorgaban, perdiendo a esos nuevos clientes. Los problemas logísticos fueron las causas del fracaso de la expansión.

Pablo está sentado en su escritorio pensando cómo solucionar este problema en los próximos seis meses antes de la reunión con el dueño. Él necesita que Simplemail logre mayor fidelización y rentabilidad, pero se pregunta: ¿Qué actividades pueden estar generando problemas de eficiencia y cómo podemos medir y solucionar esto?

\section{Historia de Simplemail}

El 12 de julio de 1995 nace Simplemail bajo el liderazgo de Pablo Rodríguez, de profesión contador, quien luego de haber trabajado para Correos de Chile decidió emprender en el rubro de la correspondencia, y citando al empresario: "Vi un nicho en el mercado, un nicho que no estaba siendo atendido, un nicho en donde las empresas querían tener a alguien fiel y responsable a su lado, un nicho que buscaba la personalización en el servicio". Así fue como Simplemail comenzó, en donde Pablo integró a la familia (madre, hermano y cuñado) al negocio.

Así, luego de incluir a sus parientes a la empresa familiar, se comienza con el proceso de contratación de motociclistas, quienes son fundamentales para la ejecución de las tareas diarias. Con el tiempo han logrado mejorar sus actividades y poder desempeñarse en el día a día. 
Con el tiempo Simplemail logró tener 4 clientes en julio de 2013, los que están felices y totalmente fidelizados. Esto gracias a la personalización del servicio y despacho regularmente bueno.

\section{Modelo de negocio Simplemail}

\section{A. $\quad$ Servicio ofrecido}

La empresa ofrecía los servicios de entrega de facturas, envío de documentos según su peso, servicio con entrega urgente y entregas en Buin, Paine y Talagante, cada uno con una tarifa específica (ver anexo 1).

Simplemail según su CEO: "Se identifica como una compañía que brindaba un servicio personalizado y diferenciado a sus clientes". El proceso se iniciaba con el retiro de la correspondencia a una hora y fecha estipuladas, para luego preparar las rutas a los diferentes destinos. Si las facturas, notas de crédito u otros documentos venían dentro de sobres, se elaboraban listados para los clientes que incluían: nombre, ruta, firma, timbre y fecha. Si se les incluía una cuarta copia (cedible, la que certificaba que el servicio se había realizado), eran debidamente firmados y entregados dentro de las 72 horas siguientes concluido el servicio. Si no se encontraban moradores en el lugar de entrega, se devolvían los documentos enviando una foto del lugar vía mensaje de texto por teléfono móvil o correo electrónico para corroborar que se llegó al destino. Otro servicio se ofrecía a los clientes cuando necesitaban un envío urgente; en ese caso solo se escaneaba y se enviaba vía correo la factura correspondiente. 


\section{B. Entrega del servicio}

El radio de entrega de Simplemail cubría toda la Región Metropolitana de Chile, alcanzando áreas hacia el norte hasta el km 22 de Panamericana, hacia el sur, km 25 de Panamericana, hacia el oriente por Av. Las Condes hasta altura $15.000 \mathrm{y}$ hacia el poniente sus entregas llegaban al límite de Pudahuel y Quilicura (ver anexo 2).

\section{Actividades primarias}

Primero, los insumos que ingresaban para realizar los procesos eran tinta y hojas, los que se estimaban para ver qué cantidad sería necesaria de acuerdo con los pedidos de los clientes. En segundo lugar estaba el ingreso de información. Cuando el cliente compraba el servicio entraba un gran número de información que era traspasada manualmente. En cuanto a la logística de salida, existía un almacenamiento de las encomiendas que se efectuaba en la oficina de Simplemail. Estas eran distribuidas por los carteros siguiendo la ruta que se les asignaba. Era importante que el cartero certificara la entrega del documento al cliente, ya que así se evitaban problemas posteriores.

Las operaciones de la empresa se basaban principalmente en la entrega de las encomiendas, facturas y pedidos a los distintos clientes. Existían distintas rutas a seguir, distribuidas según cliente. Posterior a la entrega de los distintos documentos se realizaba una revisión de que los documentos entregados y recibidos fueran los correctos y en los tiempos determinados.

Según Pablo, "el marketing no era prioridad debido a la estabilidad en su negocio". Sin embargo, se llevaba a cabo un business to business (B2B), en el que el empresario buscaba empresas que pudieran necesitar de sus servicios y de esta forma cerrar contratos. Debido a que Simplemail se había ganado una 
buena reputación por la entrega de un servicio personalizado, existía un efecto de boca en boca y habían acudido otras empresas que querían contratar sus servicios. El contacto con potenciales clientes era mediante cartas de presentación, carpetas y sitio web.

Por último, los servicios ofrecidos postentrega variaban según la urgencia que el cliente contrataba. Debido a lo anterior se generaban planes para los siguientes tres días para solucionar los distintos problemas. En el caso de que existiesen problemas como la no entrega de un documento, la empresa consideraba urgente solucionar dicho problema. Esto para mantener su línea de diferenciación del servicio.

\section{Integración horizontal}

Para Simplemail, la logística de entrada, operaciones y de salida eran fundamentales para un óptimo desarrollo de la empresa. Esta tiene una relación directa con los recursos humanos, pues eran quienes realizaban los traslados de las encomiendas $\mathrm{y}$ era sumamente importante poder potenciar algún sistema para retener a los carteros, ya que la tasa de rotación es sumamente elevada ${ }^{1}$ (cada seis meses se va algún cartero), lo que implicaba elevar sus costos. A la vez estas tres actividades estaban relacionadas con su infraestructura, en donde día a día se analizaban los pedidos y las rutas por las cuales había que realizar la entrega.

No existía un vínculo muy desarrollado entre las principales actividades de Simplemail (logística de entrada, operaciones y de salida) con el desarrollo tecnológico, lo que era uno de los motivos que generaba una ineficiencia en los procesos, debido a que toda la información que la empresa tenía era trabajada de forma manual, generando de esta forma un elevado costo en tiempo, que según

\footnotetext{
${ }^{1}$ Palabras de Pablo Rodríguez.
} 
Pablo, podía ser utilizado en la planificación estratégica de mediano plazo.

Todas las actividades de Simplemail estaban enfocadas en entregar un servicio personalizado, siendo este diferenciado en comparación a sus competidores. Por tanto existía una coherencia interna entre estas logrando un objetivo común (servicio diferenciado).

"Simplemail cuenta con un buen sistema de entrega y fabricación de facturas, además de rutas bien definidas y usos de motocicletas que minimizan el tiempo de traslado. En contraparte hay ineficiencias en el desarrollo tecnológico, siendo uno de los principales impedimentos para la expansión",2.

\section{E. Funcionamiento general de un pedido}

Las actividades de Simplemail comenzaban con el ingreso del pedido del cliente, en donde se debía identificar si era un cliente antiguo o nuevo para ver si existían precios prioritarios, descuentos por fidelidad a la empresa o preferencias especiales (ver anexo 3). Si el cliente no era nuevo, se creaba su perfil y luego se buscaba en la base de datos la información necesaria para hacer el pedido. Luego de identificar al cliente, Simplemail ingresaba el pedido, donde la empresa le definía el tipo de pedido para luego identificar el precio asociado de este. Esos datos eran ingresados a una base de datos en la que se les asignaba a un motorista y una ruta. Esta era definida a base de rutas predeterminadas. Luego que el pedido salía, llegaba a su destino en donde podía o no ser entregado, dependiendo si cumplía con los requisitos de entrega: solo puede ser recibido y firmado por la persona que se indicó que recibiría la entrega; si no

\footnotetext{
${ }^{2}$ Palabras de Pablo Rodríguez.
} 
se encuentra, la entrega volvía a la empresa. Finalmente volvía la factura final a la empresa donde era revisada e ingresada para ver si el producto fue entregado. Si no fue así, se hacía el proceso de entrega nuevamente.

\section{F. $\quad$ Actividades secundarias}

Simplemail contaba con activos tecnológicos como computadores de alta tecnología e impresoras que apoyaban el trabajo a realizar, pero a pesar de tener estos activos existía la falta de espacios para poder mecanizar los procesos y de esta forma generar economías de escala. Los recursos humanos se consideraban la fuente de valor de la empresa, ya que se necesitaban carteros para llevar los pedidos. Otro recurso humano importante eran las personas que trabajaban en la oficina misma.

Por otra parte, "el desarrollo tecnológico es un factor a considerar dentro de este negocio, siendo este punto una de las principales falencias que hoy posee Simplemail" "3. Los procesos de archivar y analizar información se realizaban de forma manual, lo que llevaba mucho tiempo. Además, no contaba con correos corporativos que ayudaran a la comunicación entre los trabajadores. Otro factor importante era que los clientes de Simplemail querían saber siempre en qué proceso iba su pedido. Por último, según Pablo, otra falencia que existía era que "aunque la página web existe, tiene que ser mejorada entregando información relevante al cliente, como los precios".

Al 15 de junio la empresa no contaba con motos propias, sino que eran de los propios carteros. Lo anterior tenía el objetivo de que cuidaran de su vehículo.

\footnotetext{
${ }^{3}$ Palabras de Pablo Rodríguez.
} 


\section{Recursos y actividades de Simplemail}

\section{A. $\quad$ Planeación estratégica}

Simplemail al 15 de junio operaba bajo una estrategia de diferenciación de servicio. Sin embargo su capacidad productiva estaba saturada. Por ello no contaba con un plan de crecimiento de la cartera de clientes, sino que vivía el día a día preocupada por gestionar sus procesos para minimizar tiempos de entrega que le permitieran en un momento dado planear. La empresa carecía de metas claras.

Dentro de las motivaciones de los trabajadores siempre existían objetivos de corto, mediano y largo plazo, lo que se veía reflejado en las metas que proponía Pablo en los distintos plazos. Esto le permitía mantener motivados a sus trabajadores. Por ello la empresa buscaba que se sintieran identificados con Simplemail, para poder transmitir el mensaje que buscaba entregar a sus clientes. También se buscaba generar compromiso por parte del empleado, para que se pudieran establecer metas personales con la empresa, como el cumplimiento de sus entregas diarias, semanales y mensuales, lo que le permitiría en un mediano plazo mejorar sus condiciones laborales. "Simplemail contaba con uniformes corporativos tanto para administración y motoristas, lo que brindaba un sello de calidad y compromiso, mejorando la imagen de la empresa"4.

\section{B. Sistemas de información}

Simplemail se encargaba de procesar todos los datos, facturas y boletas de forma manual. Esta información después era capturada en un archivo Excel. "Esto ha generado complejidad para un apto

\footnotetext{
${ }^{4}$ Palabras de Pablo Rodríguez.
} 
funcionamiento debido a la demora que toma analizar a cada cliente. Es importante para la optimización del tiempo en servicios de entrega un buen sistema de información que permita agrupar, ordenar y facilitar el análisis de datos que existen y existirán”, como declaró Pablo Rodríguez.

Por otra parte, es importante que Simplemail al entregar un servicio personalizado maneje una página web profesional (ver anexo 4), excluyendo errores que le permitan generar en sus clientes una mala imagen. El servicio web ofrecía a sus clientes dar seguimiento a la entrega de sus documentos para saber en qué etapa del servicio se encontraba. Este factor es una ventaja que Simplemail debe mantener debido a que es un atractivo tangible hacia sus clientes.

Como bien dijo Pablo Rodríguez, "La imagen que actualmente se están haciendo los clientes al ingresar a la página web no está acorde con lo que quiere la empresa debido a algunos problemas que se presentan como la usabilidad de esta, dando baja impresión de que se trata de una empresa de correos". Mejorando lo anterior, Simplemail podrá proyectar una imagen de marca más potente mostrándose como un servicio más profesional. Es importante realizar esfuerzos en la arquitectura de la página web de Simplemail.

Pablo y Javier dedicaban el $90 \%$ de su tiempo a ingresar documentos y facturas, el $10 \%$ restante contactaban clientes. "No nos queda tiempo para poder expandir nuestra cartera de clientes, tuvimos muchas facturas, lo que hacía imposible enfocarse en la captación”.

\section{Imagen corporativa}

Simplemail tenía como objetivo posicionarse como una empresa de muy alta calidad, por lo que era primordial tener un sello como empresa. Por ello, personal tanto del área administrativa como de 
entrega contaban con un uniforme a la altura de cualquier compañía mayor dentro del rubro, con claro énfasis en el logotipo y el eslogan.

Las motocicletas tenían un sello de la empresa en las cajas donde se llevaba el producto a entregar para hacer publicidad en las calles y reflejar la imagen corporativa en las mismas rutas de entrega.

\section{Recursos humanos}

Simplemail contaba con siete empleados, entre ellos su fundador Pablo Rodríguez. El resto de los trabajadores se dividían en: cuatro en el área de entrega y otros dos en el área administrativa junto con Pablo. Las funciones del área administrativa era hacer funcionar todo lo contable, adquisiciones, comercial y captación de clientes. Por otro lado, el área de entrega se dedicaba netamente a entregar el producto de manera eficiente. Cada motorista tenía asignada un área de trabajo dentro de Santiago que abarcaba ciertas comunas.

Simplemail ofrecía a los motoristas un sueldo mayor que el mercado (ver anexo 5). Sin embargo, su rotación de empleados era de aproximadamente $25 \%^{5}$ cada ocho meses. Esto provocaba episodios en los que no se había entregado el producto debido a la renuncia de algún empleado de cierta área, lo que le imposibilitaba seguir operando de manera normal en ese segmento durante un tiempo. La razón de estas renuncias era por las pocas oportunidades de crecimiento que tenían dentro de la empresa. Esta alta rotación provocaba ineficiencia del servicio de entrega por tener un nuevo empleado operando sin conocer las rutas.

La empresa también tenía problemas al entregar el producto cuando una motocicleta fallaba, pues no era posible seguir operando dentro de ese sector al no contar con una motocicleta de repuesto. Las motocicletas eran facilitadas por los mismos empleados,

\footnotetext{
${ }^{5}$ Solo se considera los motoristas.
} 
Simplemail exigía esta condición para poder trabajar en esta área de la empresa, pero al mismo tiempo desconocía el estado y condición de los vehículos. Desde el 11 de julio del 2013 la empresa facilitó motocicletas a sus empleados, pero estos abusaban de ellas, por lo que el costo de reparación era muy alto y se optó que fuera requisito fundamental para ser motorista, que cada uno tuviera su propio medio de transporte. Es por esto que la empresa debe generar un filtro con mayor rigurosidad para evitar estos costos que significa no poder operar en algún sector de entrega.

\section{E. Marketing}

Simplemail no contaba con una estrategia clara de marketing para captar y fidelizar nuevos clientes. A la vez carecía de algún tipo de marketing digital. La fidelización que realizaba la empresa era de contacto personal, mediante llamadas semanales a los clientes para preguntarles su percepción acerca del servicio brindado.

La página web presentaba errores ortográficos, secciones no habilitadas, pero a la vez contaba con complementos importantes como el seguimiento de los productos.

El marketing que llevaba a cabo Simplemail era de Worth of Mouth (WoM), es decir, buscaban clientes de manera directa. Esto generaba una inversión en tiempo para lograr identificar la satifacción de los clientes.

\section{Aires de crecimiento}

Desde 1995 Simplemail había intentado ofrecer un servicio de alta calidad para sus clientes. Su estrategia consistía en ofrecer la mayor calidad que esperaba el cliente en cuanto al servicio de espera, a un precio mayor que el mercado. Desde el 2010 la cartera de clientes aumentó para la empresa (de un cliente a cuatro clientes), por lo que 
su promesa de ofrecer el mejor servicio no fue la esperada por los clientes, fallando en entregas, encomiendas y facturaciones, es por esto que al no poder etregar la calidad necesaria pasó de tener seis clientes a solo cuatro. El desafío de Simplemail era ofrecer el servicio personalizado y prometido, lo que no se cumplía a cabalidad debido a falencias en entregas que tenían de raíz muchas otras actividades que le repercutían a este segmento del servicio tan relevante para al empresa y tan apreciado por el cliente.

Por otro lado, Pablo Rodríguez miraba de reojo abarcar nuevas áreas dentro de Chile, como la IV, V, VI y VII Región, estas implicarían un crecimiento mayor en la cartera de clientes de Simplemail. El crecimiento traía desafíos de mayor envergadura para la empresa, como la contratación de personal, la construcción de instalaciones, inversiones, entre otras. Pablo Rodríguez subrayaba su idea de crecimiento:

"Simplemail hoy en día tiene clientes conformes y fidelizados a la empresa. Nuestro desafío hoy es poder optimizar nuestros procesos más débiles, para así ser más competitivos dentro de la industria. Luego de esto, la idea es llegar fuera de Santiago, como por ejemplo Valparaíso, La Serena, Rancagua, entre muchas otras, con el objetivo de ser un agente con mayor relevancia en el mercado".

\section{Información complementaria al caso "Simplemail: modernización de procesos"}

A. La industria de las correspondencias en Chile

La industria de correspondencia tenía ciertas distinciones dependiendo del tipo de producto que se requería, siendo estos documentados y no documentados, los que diferían del tipo de 
complejidad y la forma de medir, siendo la medida en número o kilos, el que podemos ver a continuación.

a. Clasificación por tipo de productos de la industria

La industria de correos se diferencia por dos tipos de envíos, postal y CEP (Courier, Expreso y Paquetería) y además existe una subcategoría en estas aéreas, siendo productos documentados, no documentados e impresos.

En los documentados tenemos dos tipos y estos son medidos en números de envío:

1) Documentados corrientes: Se refiere a cartas personales, circulares, informes bancarios, previsionales y financieros. Estos son enviados por el medio tradicional de correo.

2) Documentados certificados: Son productos que necesitan ser certificados por el cliente. Este tipo es de mayor complejidad, por lo que está asociado a un costo mayor.

En cambio en los no documentados posee de otros dos tipos:

1) Paquetes y encomiendas: Son productos de mayor envergadura y estos son medidos en kilogramos.

2) Valija-Cliente: Se informa mediante número de valijas que el cliente entrega para su envío.

Por último se encuentran los impresos, que se refiere a diarios, revistas, folletos y otros tipos de impresos generales. Esta categoría se mide en kilogramos.

A esto Pablo añade lo siguiente: "A pesar de eso no existía mayor complejidad en el tipo de producto, sino más bien que la complejidad aparecía en la forma y tiempo de entrega, en donde la logística jugaba un rol fundamental”. 
La industria de correspondencia había sufrido una variación positiva en sus ingresos, en donde el año 2008 registró ganancias totales de $\$ 191.094 .893^{6}$ y en el $2012 \$ 237.652 .571^{7}$. Estos ingresos eran el reflejo de todos los tipos de transacciones de esta industria, registrándose documentos corrientes, certificados y valijas, como a la vez los no documentados que eran paquetesencomiendas e impresos. Todo esto podemos apreciarlo en el gráfico 1 .

\section{Gráfico 1}

Ingresos de la industria

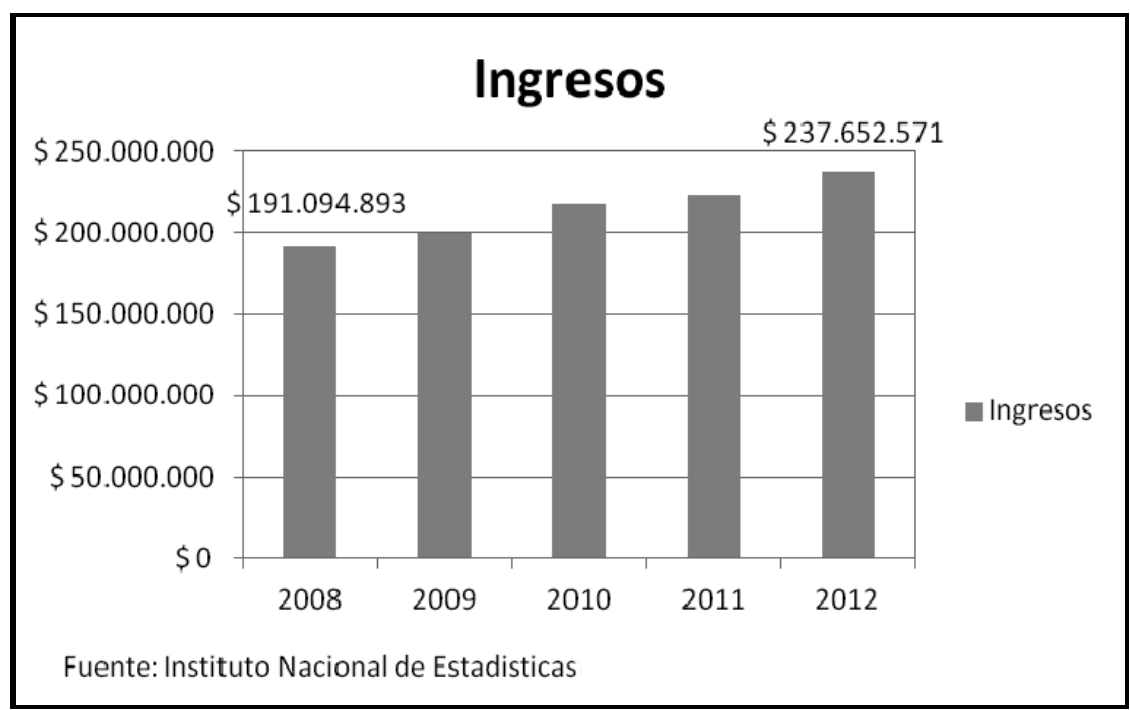

Por medio de esto podemos apreciar que en los documentados solo hubo un incremento en un tipo de producto, los certificados. En

${ }^{6}$ Los puntos indican separación de miles de pesos chilenos. 1 US es equivalente a 504,96 pesos chilenos.

${ }^{7}$ Los puntos indican separación de miles de pesos chilenos. 1 US es equivalente a 504,96 pesos chilenos. 
cambio, los corrientes sufrieron una variación desde el 2008, pero para el año 2012 el total de envíos había disminuido considerablemente. Mientras tanto los no documentados tenemos que los paquetes-encomiendas sufrieron una baja en la cantidad de kilogramos enviados en el último período. Mientras que las valijas e impresos no sufrieron grandes variaciones y se habían mantenido a lo largo de los últimos cinco años. En el gráfico 2 tenemos lo siguiente:

\section{Gráfico 2}

Variación del total de productos enviados en la Región Metropolitana

\section{Total de números de envíos locales por tipo de envío}

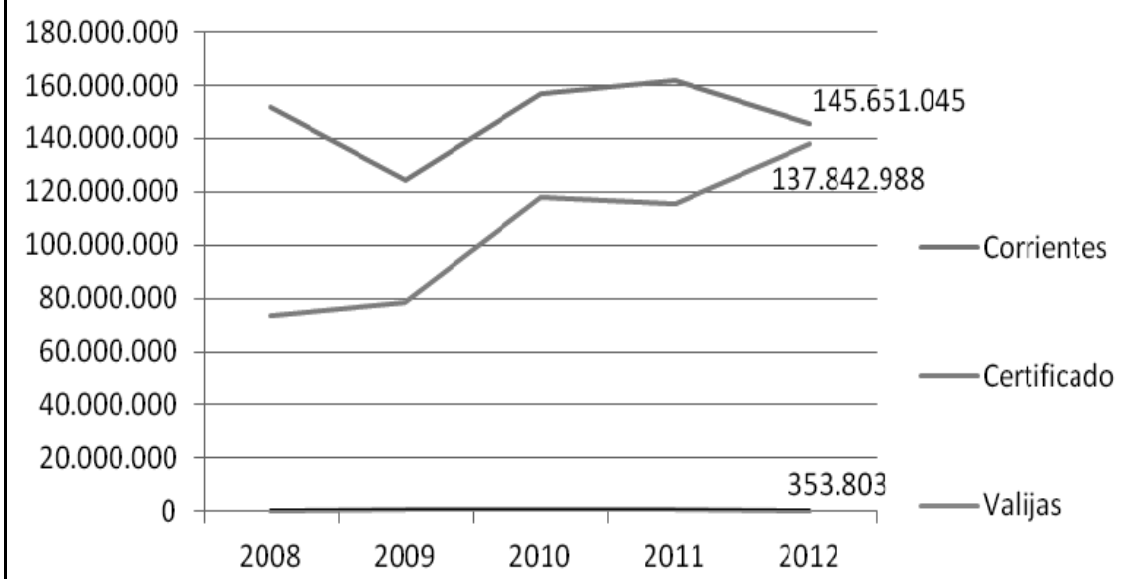

Fuente: Instituto Nacional de Estadísticas 


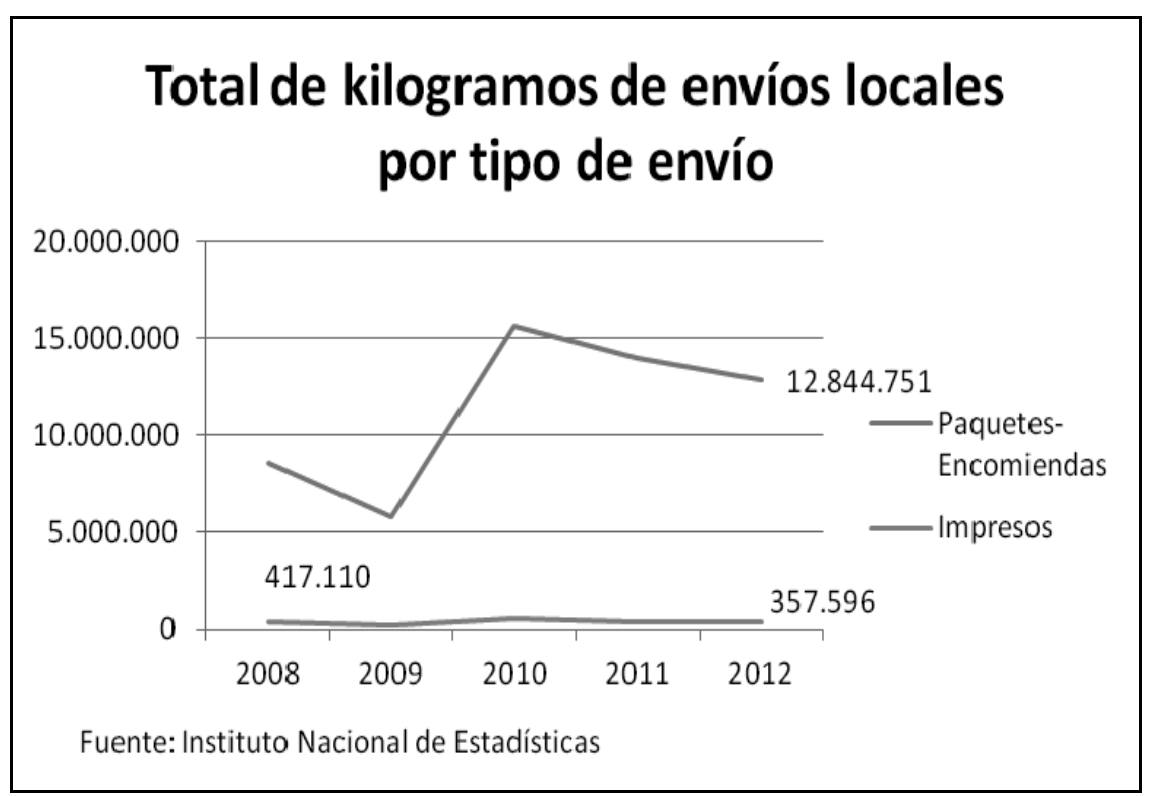

Otro aspecto relevante era el hecho de la participación de los documentos en sus tipos, en donde los paquetes-encomiendas representaban el 96\% del total de entregas de documentos medidos en kilogramos, mientras que los impresos eran 4\%. Por otro lado los documentos que eran medidos en número de envíos se encontraba una participación del 50,1\% para certificados, 49,8\% para corrientes y $0,1 \%$ para valijas. Todo esto podemos apreciarlo en el gráfico 3 . 


\section{Gráfico 3}

Participación de las cantidades enviadas en la Región Metropolitana

\section{Total de kg de envíos en la Región Metropolitana año 2012}

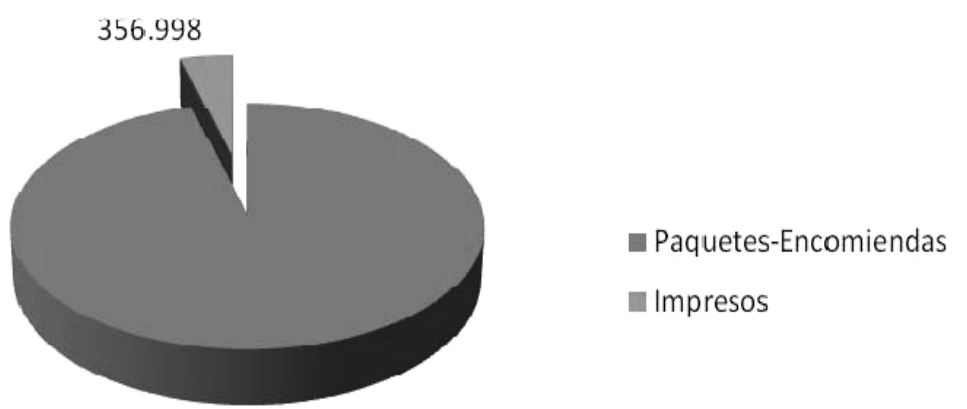

8.225 .380

Fuente: Instituto Nacional de Estadísticas

\section{Total de número de envíos en la Región Metropolitana año 2012}

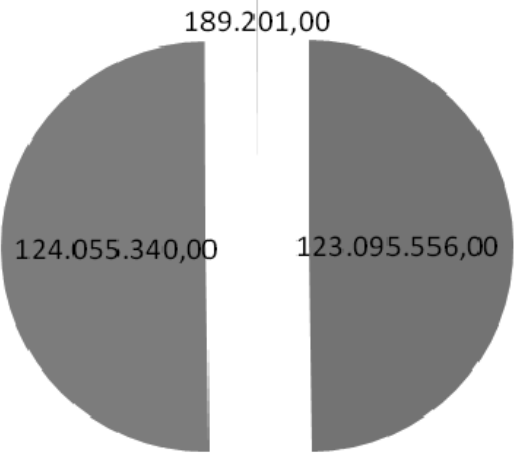

Corrientes

- Certificado

- Valijas

Fuente: Instituto Nacional de Estadísticas 
En esta industria para julio del 2013 existen grandes empresas que lideran el mercado chileno: Fedex, Correos de Chile y Chile Expres. A la vez las empresas se dedicaban a los negocios de B2B (Business to business) y B2C (Business to Consumer).

Correos de Chile registraba ingresos de 77 mil millones de pesos en el 2012. En donde desde el 2011 hasta el 2012 hubo un incremento de las ventas en mercados institucionales como sucursales ( $9 \%$ y $4 \%$, respectivamente). Correos de Chile tiene $5.228^{8}$ trabajadores, lo que ayuda y apoya notablemente al crecimiento y servicio otorgado por ellos.

La industria se veía amenazada por la entrada, todavía no tan explosiva, de la facturación electrónica, la que facilitaría a las empresas realizar sus facturas por computadora y enviarlas a sus clientes por el mismo medio.

Los principales costos para esta industria eran la mano de obra, pues según el tipo de cliente se necesitaba más o menos personal; el combustible, cuyo costo era bajo en comparación con las utilidades; y la inversión en tecnología y activos fijos, que eran costos elevados. Y según dichos del empresario Pablo: "La tecnología es un elemento fundamental para lograr una mejor gestión en el transporte y logística, así uno puede abaratar tanto costos económicos como de tiempo".

Las empresas de esta industria eran de dos estilos, por costo o personalización. Esto es que hay organizaciones que buscan rentabilidad reduciendo sus costos y generando economías de escala y otras se enfocan en dar soluciones personalizadas a sus clientes.

\footnotetext{
${ }^{8}$ Memoria Correos de Chile año 2012.
} 


\section{Anexos}

Anexo 1

Tarifas año 2013

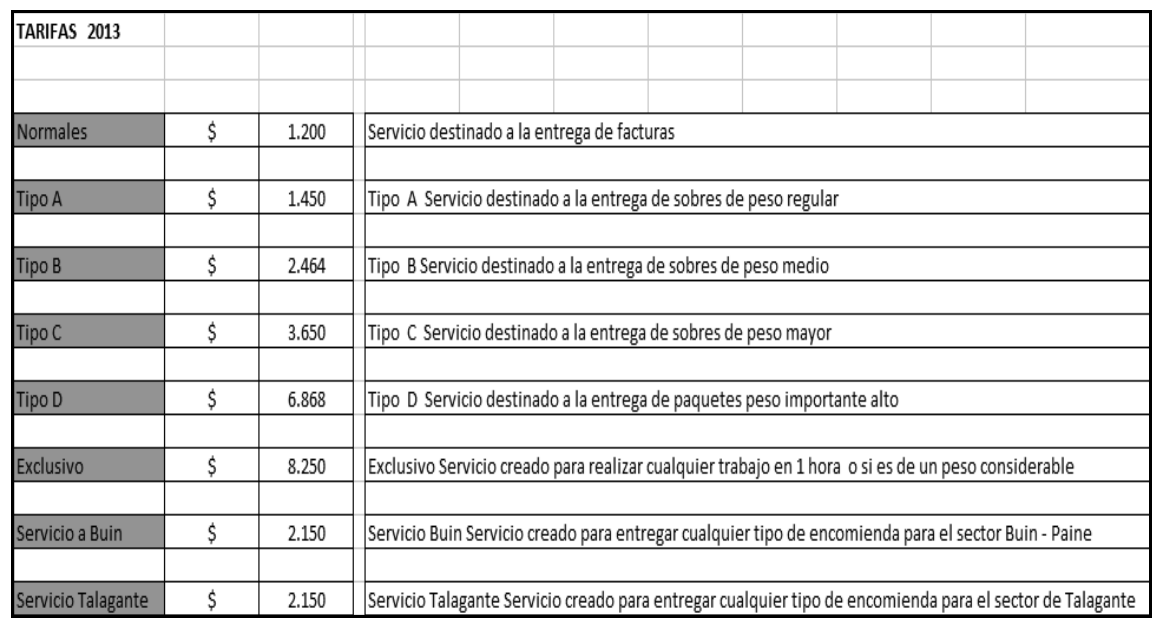

FUENTE: Simplemail 


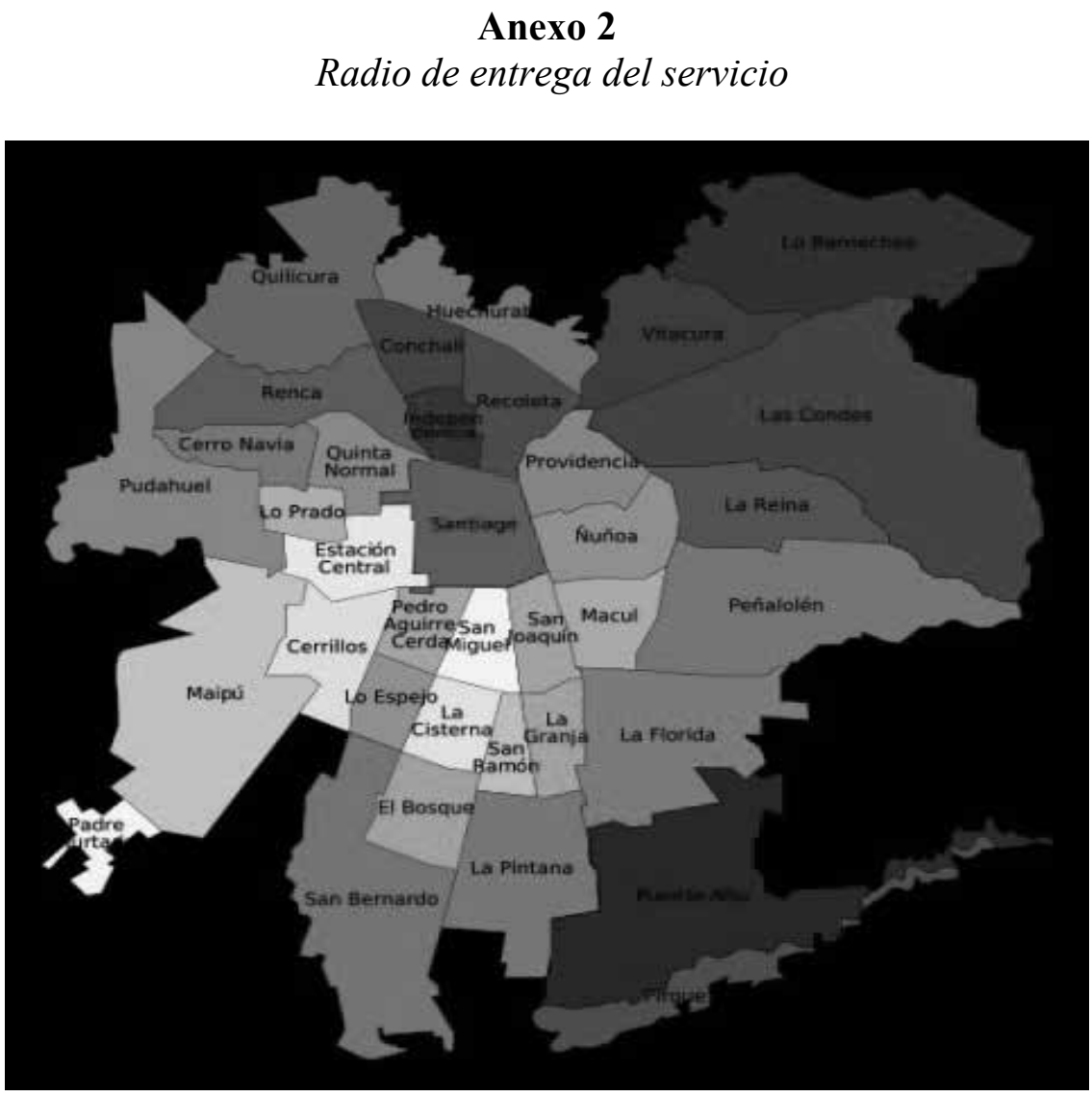

FUENTE: Mapa Simplemail. 
Anexo 3

\section{Diagrama de flujo}
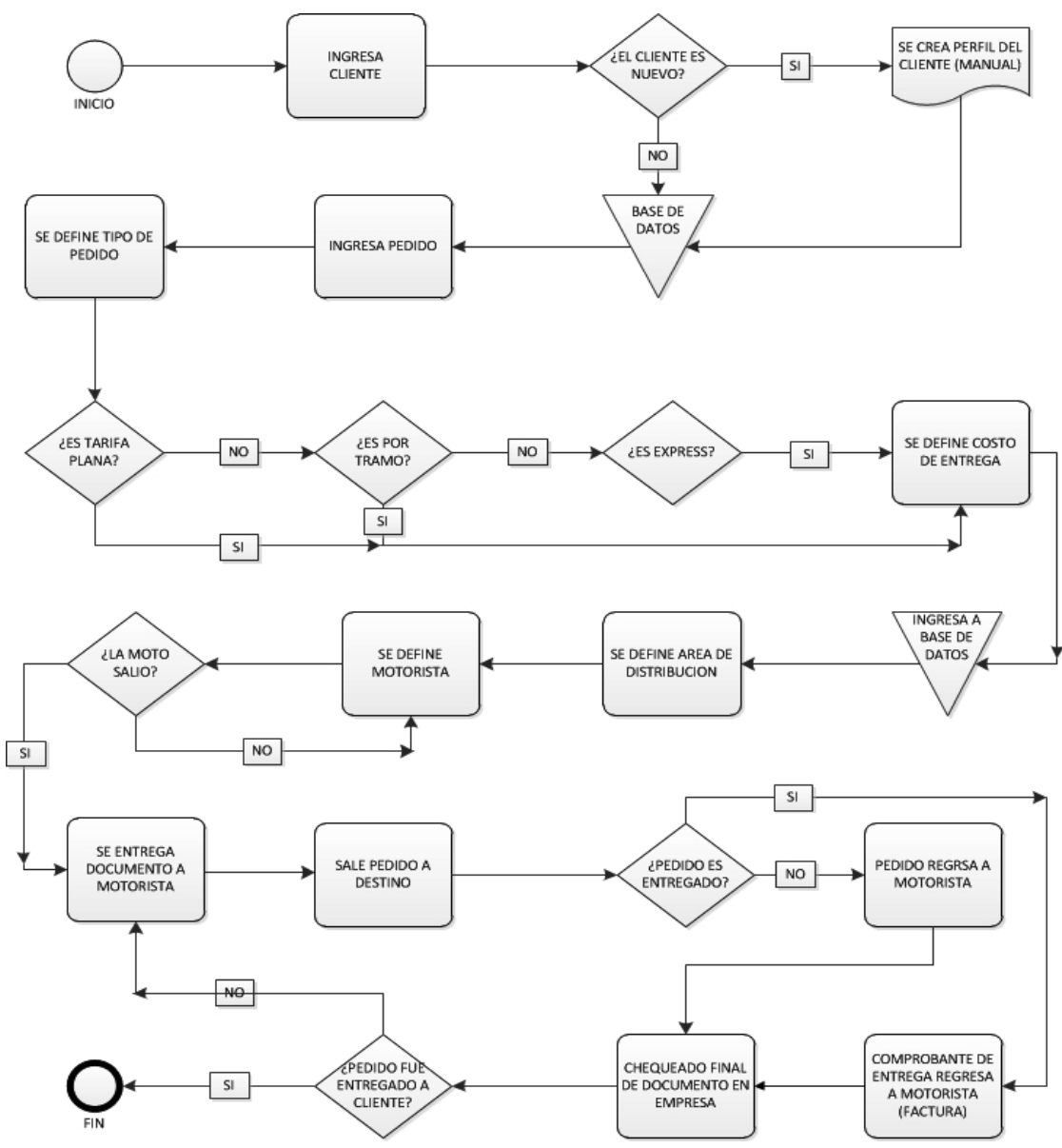

FuENTE: Autores del caso "Modernización de Proceso Simplemail": Patricio Morales, Pieter van Eijck y Juan Pablo Vidal. 


\section{Anexo 4 \\ Página web}

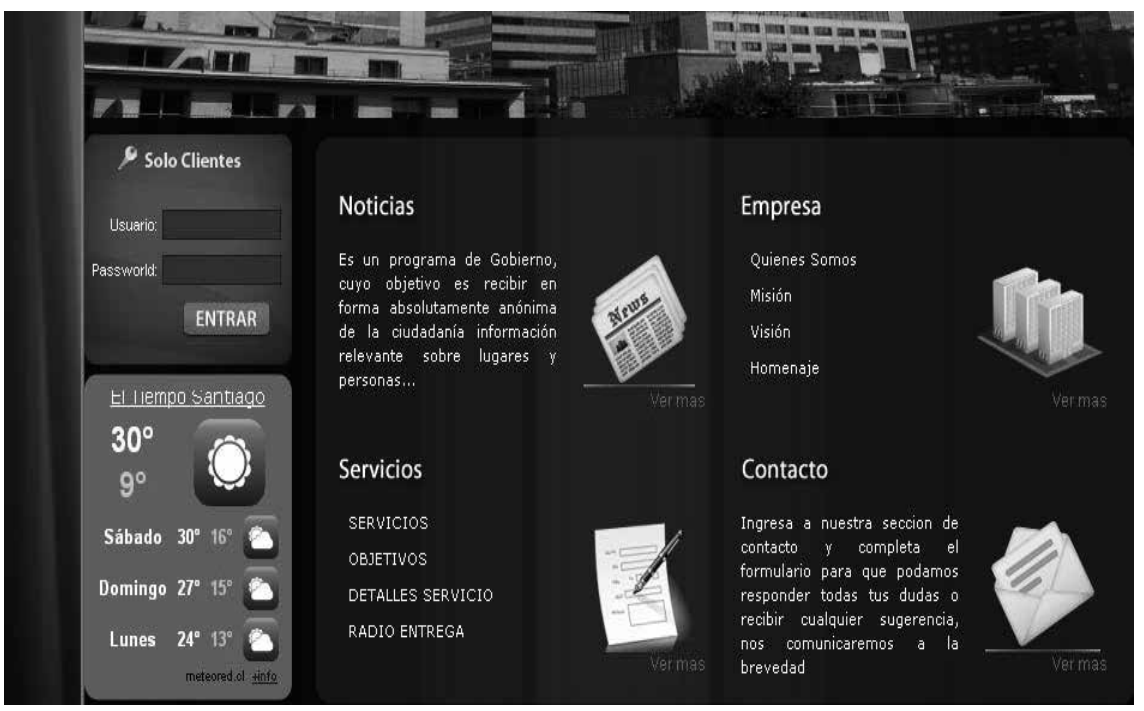


Anexo 5

Sueldos motoristas en Santiago

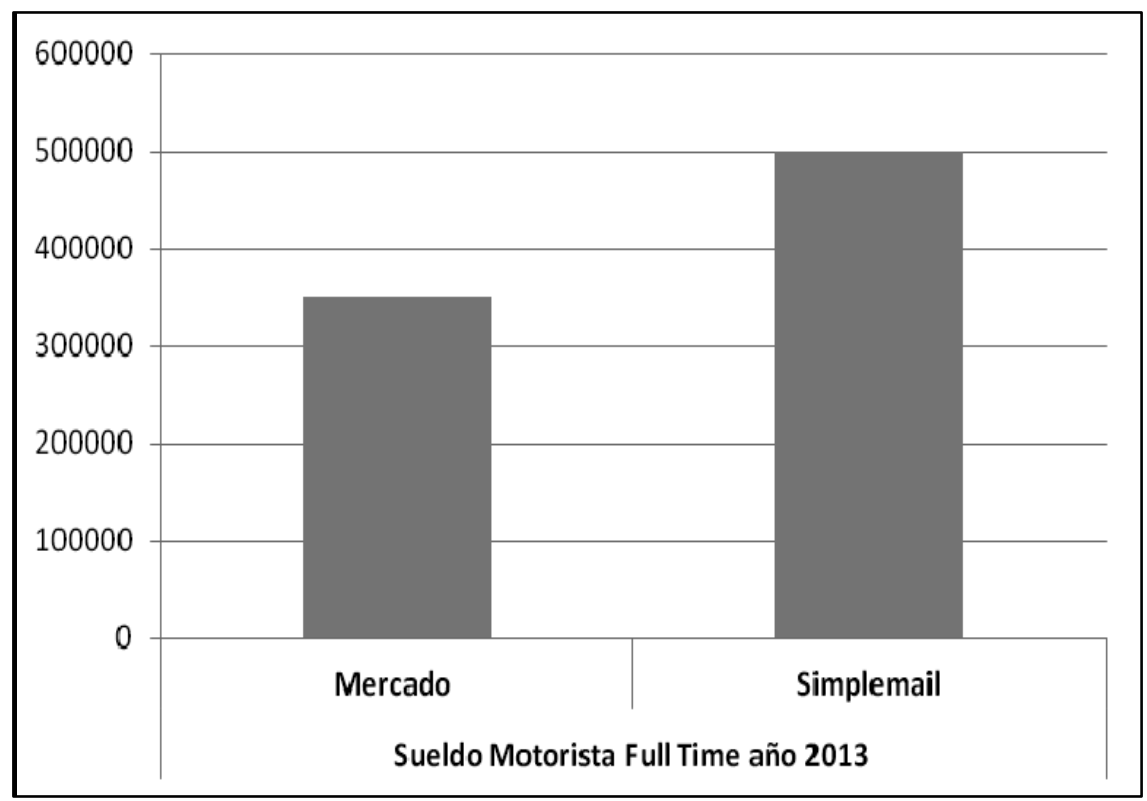

FUENTE: Simplemail 